\title{
Successful thrombolytic therapy in a patient with congenital corrected transposition of the great arteries
}

\author{
Selcuk Ozturk, Fatma Erdem, Serkan Ozturk, Selim Ayhan \\ Abant Izzet Baysal, University Izzet \\ Baysal Research and Training Hospital \\ Department of Cardiology, Bolu,Turkey \\ Correspondence: \\ selcukozturk85@hotmail.com \\ Tel.: + 905535376613 \\ Fax.: + 903123633396 \\ Received: 19 February 2016 \\ Accepted: 16 June 2016 \\ Key words: Congenitally corrected \\ transposition of the great vessels • \\ Myocardial infarction - Thrombolytic \\ therapy.

\begin{abstract}
Objective. The aim of this report is to emphasize the importance of thrombolytic therapy in selected patients, such as those with congenital heart defects in whom a coronary artery anomaly can be observed. Case report. We present here a 63 year-old female patient who was admitted to our emergency department with ST segment elevation myocardial infarction and a history of a congenital heart defect. We treated the patient successfully with thrombolytic therapy instead of primary percutaneous intervention, because of the suspicion of a coronary artery anomaly. On the following day, we performed coronary angiography on the patient, which revealed the anomalous origin of the coronary arteries, with the left and right coronary arteries originating from the right sinus of Valsalva and the circumflex artery originating from the left sinus of Valsalva. This anomaly in this patient group is described for the first time. Conclusion. Coronary artery anomaly may be observed in patients with congenitally corrected transposition of the great arteries, and in the case of requiring emergency reperfusion, thrombolytic treatment can be an alternative strategy in this patient group.
\end{abstract}

\section{Introduction}

Congenitally corrected transposition of the great arteries (ccTGA) is an unusual congenital anomally which is often diagnosed in adulthood. In ccTGA, the right atrium is connected to the morphologic left ventricle, which drains blood to the pulmonary artery, and the left atrium is linked with the morphologic right ventricle, which drains blood to the aorta (1). Its prevelance varies from approximately $0.5 \%$ to $1.4 \%$ of all congenital cardiac anomalies. Ventricular septal defect, pulmonary stenosis, atrial septal defect, situs inversus, heart blocks and arrythmias usually accompany ccTGA (2).

In ccTGA, the anatomy of the coronary arterial system is complex and the coronary arteries show a mirror-image spread. The morphological right coronary artery (RCA) originates from the left posterior sinus and the morphological left main coronary artery originates from the right anterior sinus (3). The possibility of progression of coronary artery disease is low in congenitally corrected TGA patients because these patients do not survive to old age due to the existing comorbidities (3). In the medical literature there is no evidence of any patient with ccTGA and ST segment elevation myocardial infarction (STEMI) which needs emergency reperfusion treatment. In this report, we describe a case of a ccTGA and STEMI patient, whom we treated successfully with thrombolytic therapy and in whom we detected 
a coronary artery anomaly in the coronary angiography, which has not been mentioned in this patient group previously.

\section{Case report}

A 63 year-old female patient presented in the emergency room complaining of chest pain which had started three hours before. She had no risk factors for coronary artery disease, except mild hypercholesterolemia. Physical examination revealed a heart rate of 96 beats/min, blood pressure of 110/80 mmHg, S3 gallop with S1, S2 and 3/6 systolic murmur in the cardiac apex. Electrocardiography (ECG) showed ST segment elevation in V 1-4 and depression in the reciprocal leads (Figure 1A).

Initial laboratory analyses revealed the following: troponin I was $0.9 \mathrm{ng} / \mathrm{ml}$ (normal range: $0-0.06 \mathrm{ng} / \mathrm{ml}$ ) and the creatine kinase$\mathrm{MB}$ was $85.7 \mathrm{U} / \mathrm{l}$ (normal range: 0-24 U/1). The remaining blood count and biochemistry results were within normal ranges. It was learned from the patient's history that she had a congenital cardiac anomaly, but she had no report or hospital records confirming this condition. The patient received 300 $\mathrm{mg}$ acetylsalicylic acid, $300 \mathrm{mg}$ clopidogrel orally and $5000 \mathrm{U}$ unfractionated heparin intravenously, and she was transferred to the coronary intensive care unit. A bedside transthoracic echocardiography was performed, and transposition of the aorta and the pulmonary artery with transposition of the right and left ventricles were shown. The ejection fraction of the morphological right ventricle was slightly depressed (45\%) and the apical region was observed to be hypokinetic. Due to the suspicion of a coronary artery anomaly, thrombolytic therapy was planned for the patient instead of primary percutaneous coronary intervention. After receiving the patient's consent, thrombolytic therapy (alteplase) was applied. Thirty minutes after the initiation of the thrombolytic therapy, the patient's chest pain improved, and fifteen minutes later resolution of ST segment changes on ECG was observed (Figure 1B). Thrombolytic therapy was considered to have been successful. The patient remained hemodynamically stable with aspirin, clopidogrel, metoprolol, ramipril, atorvastatin and heparine treatment, and on the next day she was referred to the catheterization laboratory. Coronary angiography revealed the anomalous origin of the coronary arteries, with the left (LAD) and right (RCA) coronary arteries originating from the right sinus of Valsalva, and the circumflex artery (CX) originating from the left sinus of Valsalva. LAD was thought to

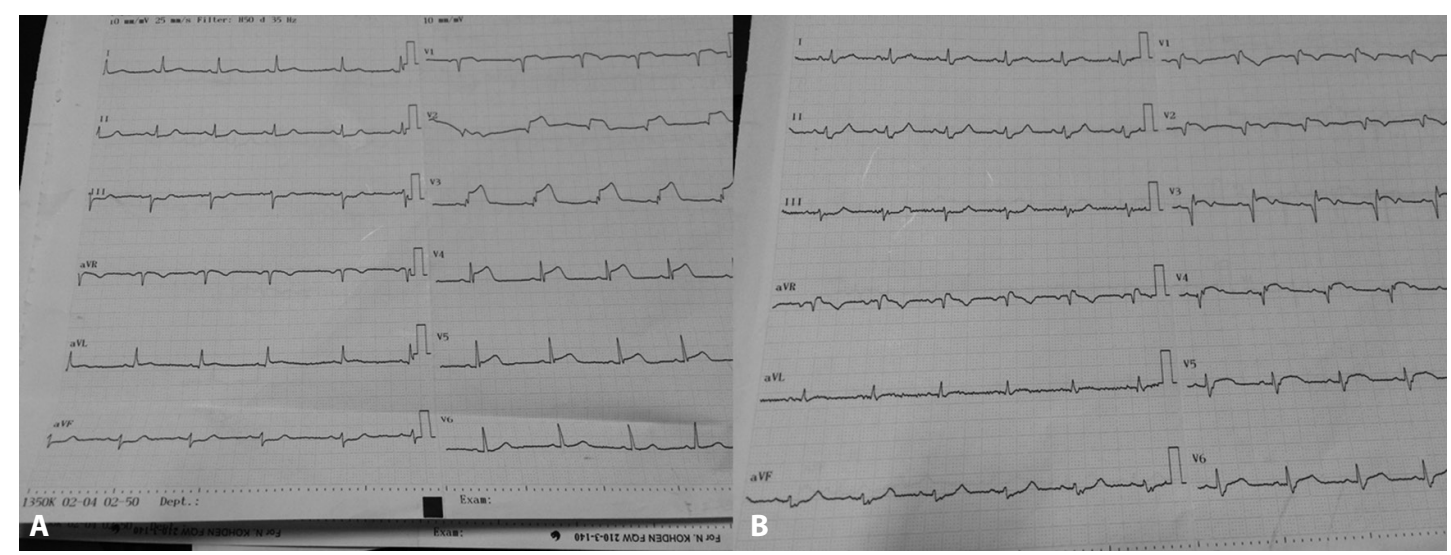

Figure $1 \mathrm{~A}$ : ECG of the patient on admission showing ST segment elevation in V1-4 with Pardee curves and depression in reciprocal leads. B: ECG of the patient showing succesful thyrombolytic treatment. 


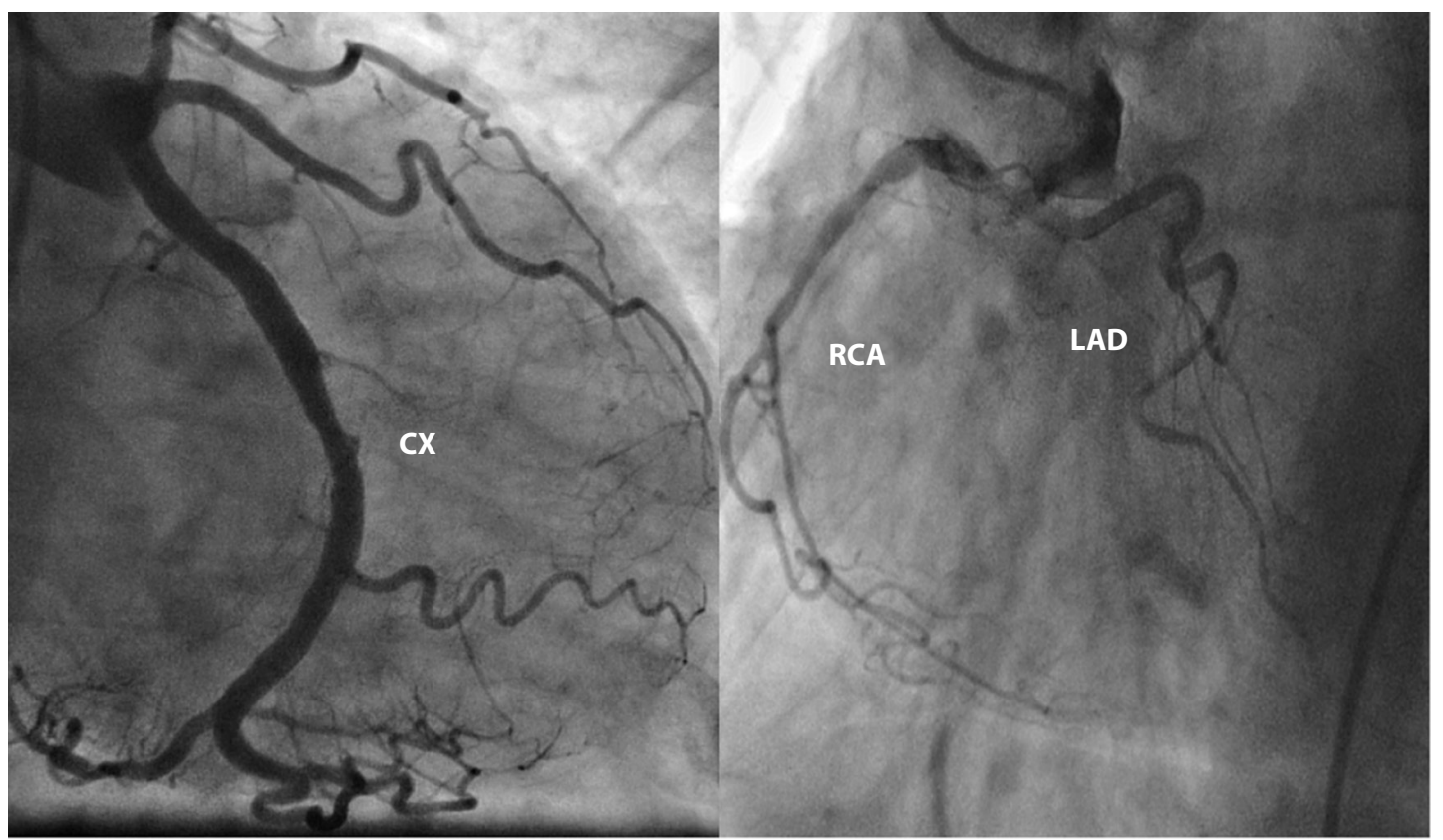

Figure 2 Coronary angiography showing the circumflex artery $(C X)$ originating from the left sinus of valsalva and right (RCA) and left (LAD) coronary arteries originating from the right sinus of valsalva. There is plaque in all the coronary arteries, which are free of hemodynamically significant stenoses.

be the culprit vessel causing anterior wall STEMI which was free of any significant obstruction after successful thrombolysis and it was decided to be followed up with medical treatment. There was plaque in all of the coronary arteries, which were free of hemodynamically significant stenoses (Figure 2).

The patient remained under observation in the cardiology service after the coronary angiography. Follow-up ECG showed normal sinus rhythm with the invertion of $\mathrm{T}$ waves in V 1-4. The follow-up echocardiogram was consistent with the bed side echocardiography performed in the coronary intensive care unit, demonstrating ccTGA. Moreover, there were no findings of complications such as ventricular septal defect or aneurysm of the morphological right ventricle. Follow-up laboratory tests also revealed a peak in the cardiac enzymes (troponin I >25 ng/ml, creatine kinase-MB: 108.3 $\mathrm{U} / \mathrm{l})$. The patient's condition continued to be asymptomatic and stable, and she was discharged home after 3 days.

\section{Discussion}

To our knowledge, there are no published data regarding a ccTGA patient with STEMI successfully treated with thrombolytic therapy. There is also no record of a coronary artery anomaly showing the circumflex artery originating from the left sinus of Valsalva and the right and left coronary arteries originating from the right sinus of Valsalva in this patient group.

Cardiovascular diseases are the main cause of death all around the world, and their cause is the occlusion of the blood vessels with a blood clot or thrombus. Restoring the blood flow immediately can save organ functions and lives $(4,5)$. Although thrombolytic therapy has been used for years for this reason, its adequacy and reliability have been inadequate because of the side effects and failure of the therapy. Consequently, catheter guided endovascular procedures, such as percutaneous coronary intervention (PCI), have been the preferred treatment 
modality for clinicians instead of thrombolysis. Although endovascular procedures are more successful and safer than thrombolysis, there are some limitations of this treatment modality. Some of them are the technical difficulties and cost, they are time-consuming and cannot be used in cases of insufficient access (4). Although primary PCI is not contraindicated in congenital heart defect patients presenting with STEMI, and it is recommended therapy according to the guidelines, we preferred thrombolytic therapy instead of endovascular procedures because we believed thrombolysis to be the more appropriate treatment in this patient. Additionally, our patient did not have any contraindications for thrombolytic therapy, such as known bleeding disorders, history of ischaemic stroke, intracranial haemorrhage, gastrointestinal bleeding, trauma or injury, and she was admitted to hospital in three hours after the chest pain started which increases the possibility of successful reperfusion with thrombolytic therapy. Although we could have preferred primary PCI as the first line therapy in this patient, we chose thrombolytic therapy due to the suspicion of a coronary artery anomaly which could cause time to be wasted, technical difficulties and complications during PCI. Thus, after successful reperfusion with thrombolytic regimen, we proved the presence of a coronary artery anomaly in the catheterization laboratory. Haemodynamically non-significant stenoses were observed incidentally during the coronary angiography, for which the patient did not need further processing, such as angioplasty or stenting procedure.

The main goal of therapy in acute myocardial infarction is to ensure the blood flow to the myocardial cells as soon as possible, and thrombolytic therapy is one of the treatment strategies (5). However, in the literature there is no report supporting the use of thrombolytic agents in the presence of a suspected coronary artery anomaly, and there are very little data on the combination of ccTGA patients and acute coronary syndromes. Lampropoulos et al. described a case of an acute coronary syndrome and a ccTGA patient, in whom they performed coronary angiography. The angiogram of the patient showed haemodynamically nonsignificant stenoses, and they suspected that the cardiac enzyme increase was secondary to microvascular ischemia or hypertrophy (6). Gungor et al. described a case of a patient with a history of coronary artery disease who complained of unstable anginal chest pain and cardiac enzyme elevation. They planned an early invasive procedure on the patient because of the acute coronary syndrome and diagnosed ccTGA during and after the coronary angiography (7). In our case, the ccTGA patient was complaining of chest pain and she had ST segment elevation on ECG, which needed emergency reperfusion treatment, so we treated the patient successfully with thrombolytic treatment. We selected thrombolytic treatment due to the possibility of a coronary artery anomaly and, to the best of our knowledge, this is the first ccTGA patient presenting with STEMI, successfully treated with thrombolytic treatment in the literature. In this case, we also demonstrated the circumflex artery originating from the left sinus of Valsalva and the right and left coronary arteries originating from the right sinus of Valsalva, which is a coronary artery anomaly not previously mentioned in this patient group.

\section{Conclusion}

A coronary artery anomaly may be observed in ccTGA patients and in the case of the need for emergency reperfusion, thrombolytic treatment can be an alternative treatment modality in this patient group. 


\section{What is already known on this topic}

Congenitally corrected transposition of the great arteries is a rare cardiac anomaly which is characterized by atrioventricular and ventriculoarterial discordance. Acute coronary syndomes are described very rarely in patients with congenitally corrected transposition of the great arteries. Treatment of myocardial infarction with thrombolytic therapy is an alternative strategy in selected patients, instead of primary percutaneous coronary intervention.

\section{What this article adds}

This is the first case report describing a patient with congenital$l y$ corrected transposition of the great arteries and ST segment elevation myocardial infarction who was treated with thombolytic therapy. This is the first case report describing a patient with congenitally corrected transposition of the great arteries and coronary artery anomaly in which the left and right coronary arteries originate from the right sinus of Valsalva and the circumflex artery originates from the left sinus of the valsalva. In patients suspected of having a coronary artery anomaly who need emergency reperfusion treatment, thrombolytic treatment can be a good choice.

Authors' contributions: Conception and design: SO, $\mathrm{FE}$ and SO; Acquisition, analysis and interpretation of data: SO, FE and SA; Drafting the article: SO, SO, FE and SA; Revising it critically for important intellectual content: SA, FE and SO; Approved final version of the manuscript: SO.

Conflict of interest: The authors declare that they have no conflict of interest.

\section{References}

1. Lee SR, Schwartz RS, Jin GY, Ko JK. Myocardial bridge in congenitally corrected transposition of great arteries. J Cardiovasc Comput Tomogr. 2008;2(5):339-40.

2. Kaya A, Tanboga IH, Kurt M, Isık T, Ozgokce M, Topcu S, et al. Corrected transposition of the great arteries with previously unreported cardiac anomalies. Cardiovasc J Afr. 2012;23(5):5-7.

3. Kantarci M, Koplay M, Bayraktutan U, Gundogdu F, Ceviz N. Congenitally corrected transposition of the great arteries: MDCT angiography findings and interpretation of complex coronary anatomy. Int J Cardiovasc Imaging. 2007;23(3):405-10.

4. Gurewich V. Thrombolysis: A Critical First-Line Therapy with an Unfulfilled Potential. Am J Med. 2016;129(6):573-5.

5. Gurman P, Miranda OR, Nathan A, Washington C, Rosen Y, Elman NM. Recombinant tissue plasminogen activators (rtPA): a review. Clin Pharmacol Ther. 2015;97(3):274-85.

6. Lampropoulos KM, Kotsas D, Iliopoulos TA. Acute coronary syndrome in congenitally corrected transposition of the great arteries. BMJ Case Rep. 2013; 2013:bcr2012008354.

7. Gungor B, Gurkan U, Yllmaz H, Bolca O. Late diagnosis of corrected transposition of the great arteries in an elderly patient with coronary artery disease. Turk Kardiyol Dern Ars. 2012;40(1):66-8. 